\title{
«Happiness Management»: Revisión de literatura científica en el marco de la felicidad en el trabajo
}

\section{"Happiness Management»: Review of scientific literature in the framework of happiness at work}

Dr. Juan Francisco Sánchez-Vázquez es profesor e investigador de la Universidad Pontificia de Salamanca (España) (jfsanchezva@upsa.es) (http://orcid.org/0000-0002-3759-6245)

Dra. Raquel Sánchez-Ordóñez es profesora e investigadora de la Universidad Pontificia de Salamanca (España) (rsanchezor@upsa.es) (http://orcid.org/0000-0003-4439-9896)

José Santiago Jimbo Días es profesor e investigador de Cuenca (Ecuador) (santiago.jimbo@ucuenca.edu.ec) (https://orcid.org/0000-0002-2823-4285)

\begin{abstract}
Resumen
Los cambios en el mundo del trabajo y de las organizaciones y su impacto en la vida de las personas han planteado una nueva forma de abordar las relaciones entre trabajo y felicidad. Se realiza un estudio bibliométrico de los trabajos que sobre economía de la felicidad se han publicado en lengua española desde el año 2000 hasta el 2018. Para ello, se utiliza el buscador EDS-Multidisciplinar (EBSCO Discovery Service), seleccionándose 95 publicaciones que tratan esta temática. Como resultado del estudio encontramos una mayor producción de abordajes teóricos sobre el tema frente a la investigación empírica. En ésta última, los trabajos son fundamentalmente analizados desde la perspectiva económica y la psicológica. La tendencia claramente creciente de la producción científica sobre felicidad y trabajo tiene dos vertientes importantes: Por un lado, el propio tiempo de grandes cambios, que hace surgir continuamente nuevos paradigmas explicativos y prescriptivos; y, por otro lado, coincide este incremento con el fin de la crisis económica. Superar y dejar atrás las preocupaciones de este gran desequilibrio del sistema económico mundial, ha vuelto a centrar el interés por hacer más productivas y humanas las empresas, preservando la salud laboral y mejorando el desarrollo personal. En este sentido se destaca la imperiosa necesidad de un abordaje multidisciplinar de esta área desde la investigación empírica, que contribuya a mejorar las relaciones entre el mundo laboral y el bienestar subjetivo de las personas.
\end{abstract}

\begin{abstract}
The changes in the world of work and organizations and their impact on people's lives have raised a new way of approaching the relationship between work and happiness. A bibliometric study is carried out of the works on the economy of happiness that have been published in Spanish between 2000 and 2018. To do so, the EDS-Multidisciplinary search engine (EBSCO Discovery Service) is used, selecting 95 publications that deal with this subject. As a result of the study we find a greater production of theoretical approaches on the subject as opposed to empirical research. In the latter, the works are fundamentally analyzed from the economic and psychological perspective. The clearly growing trend of scientific production on happiness and work has two important aspects: On the one hand, the very time of great changes, which makes emerge continuously new explanatory and prescriptive paradigms; and, on the other hand, this increase coincides with the end of the economic crisis. Overcoming and leaving behind the preoccupations of this great imbalance of the world economic system has once again focused the interest on making companies more productive and humane, preserving occupational health and improving personal development. In this sense, the urgent need for a multidisciplinary approach to this area from empirical research that contributes to improving relations between the world of work and the subjective well-being of people is highlighted.
\end{abstract}

\section{Palabras clave I keywords}

Felicidad y trabajo, bienestar subjetivo, psicología positiva, liderazgo, gestión de felicidad, organizaciones positivas y saludables, trabajo y cambio, economía de la felicidad.

Happiness and work, subjective wellbeing, positive psychology, leadership, happiness management, positive and healthy organizations, work and change, economy of happiness.

Cómo citar: Sánchez-Vázquez, J.F., y Sánchez-Ordóñez, R. (2019). «Happiness Management»: Revisión de literatura científica en el marco de la felicidad en el trabajo. Retos Revista de Ciencias de la Administración y Economía, 9(18), 259-271.

https://doi.org/10.17163/ret.n15.2018.05 


\section{Introducción}

Debido a las tendencias dinámicas que constituyen nuestra sociedad, cada cierto tiempo se revisan y cambian sus bases más estables y se producen avances importantes en su configuración. Estos saltos en la línea del progreso remueven todas las estructuras tradicionales, los valores, las instituciones $\mathrm{y}$, en definitiva, afectan de manera importante la vida de las personas.

Numerosos investigadores en los últimos tiempos han constatado que el impacto de las Tecnologías de Información y Comunicación (TIC) y las Tecnologías del Aprendizaje y del Conocimiento (TAC), junto con la globalización y otros factores sociales importantes, han situado el centro de atención del cambio en las personas (Castells, 2008). Esto es un hecho clave en el mundo del trabajo y las organizaciones, donde han sido desautorizados y removidos todos los paradigmas antiguos que caracterizaban a las empresas de éxito y que eran seguidos y prescritos para todas las organizaciones. El paradigma neoliberal, de producción capitalista, fue concebido para la población, recursos, necesidades y problemas de hace 250 años. Primaban entonces los criterios de utilidad y libertad y las estructuras potenciaban factores muy alejados del desarrollo sostenible y responsable, la solidaridad y cooperación, el altruismo y la protección social, el bienestar y satisfacción de las personas, su salud y calidad de vida organizacional, y en definitiva, su felicidad.

Esta incompatibilidad en los sistemas antiguos es insostenible ante las exigencias de los cambios. Así, se ha propiciado una importante corriente del pensamiento antropológico, económico, social y psicológico que nuclea el llamado enfoque de la «Economía de la Felicidad» (Rivas, 2015; Frey, 2018). Sobre ésta, no hay un inventor, un pionero, ni un año de nacimiento, ni una organización que pilote sus contenidos, ni estos son únicos, pero sí hay una historia en este enfoque y está jalonada de hitos sobresalientes: entre otros, la «paradoja de Easterling», el índice «Felicidad Nacional Bruta», creado por Jigme Singye, rey de Bután, al que siguieron índices alternativos al PIB recogiendo conceptos como bienestar, equidad, justicia, medio ambiente, tiempo libre, esperanza de vida, educación, compasión, solidaridad, etc.

En este sentido, resultan innumerables las contribuciones, entre ellas el Premio Nobel de Economía de 1999 a dos psicólogos (Kahneman y Tversky) y en 2001 y 2014 a economistas con aportaciones psicológicas (Akerloff y Tirole, respectivamente). Asimismo, resulta importante traer a colación la consolidación de la Psicología Positiva y la Responsabilidad Social Corporativa, el ranking «Great Place to Work», como indicador de empresas felices y la declaración, en 2010 por la ONU, del 20 de marzo como Día Mundial de la Felicidad.

Ante las exigencias de los cambios, dentro del campo de la Psicología del Trabajo y de las Organizaciones, al igual que en las otras Ciencias Sociales, el debate sobre la felicidad y lo positivo ha cobrado mucho interés (Rodríguez-Muñoz \& SanzVergel, 2011). Son ya múltiples las concreciones y modelos que tratan de evidenciar las relaciones entre felicidad y trabajo. Por ello es importante constatar como objetivo de este estudio el estado de la cuestión en la literatura en español de las investigaciones existentes sobre felicidad, con el fin de poder comprender el presente y adentrarnos a predecir el futuro sobre este campo. 
Muchas de las contribuciones recientes a este objeto de estudio recuperan la clásica doble visión hedonista y eudaimónica de la felicidad, que operan en conjunto y ponen el acento en la experiencia del bienestar subjetivo o el bienestar objetivo -aunque se añade últimamente también el bienestar social-. La ecuación pasaría por un conjunto de experiencias evaluativas (comparación de logros, éxitos y fracasos, pérdidas, en la vida), un conjunto de experiencias afectivas (emociones y estados de ánimo experimentados positivos y negativos) y un conjunto de experiencias sensoriales (placeres y dolores sentidos), pues las personas combinan en distinto grado estas experiencias (de los Ríos, 2016; De Barbieri, 2018).

La estrategia vital más consistente en el camino de la felicidad parece tener dos direcciones: evitar el malestar y conseguir un propósito, un sentido de la vida (Thivissen, 2018). En este segundo factor es donde más puede estar ubicado todo lo relacionado con la vida laboral. Schnell (tal como se citó en Retzbach, 2018), describe cuatro características del sentido de la vida: la significación -sensación de lo que se lleva a cabo importa-, la pertenencia -sentimiento de que se tiene un lugar en el mundo-, la coherencia -lo que ocurre en la vida es armónico y congruente-, y la orientación -saber los valores y objetivos que se defienden-. Otros autores incluyen también la generatividad -crear algo beneficioso para las generaciones posteriores-.

Una dirección parecida toma las aportaciones de la Psicología Positiva (Vera, 2006 y 2008), que configura cinco características: emociones positivas, entrega, interés, sentido y propósito (Seligman, 2011). En última instancia felicidad y bienestar subjetivo van de la mano (Diener, 2000; Popescu, 2016). Y para el trabajo y las organizaciones, la clave de esta propuesta es el crecimiento personal, añadiendo autoestima, optimismo, resiliencia, vitalidad, autodeterminación y relaciones positivas.

Diferentes modelos aportan a este campo distintas concreciones (Bakker, Rodríguez-Muñoz \& Derks, 2012). Por ejemplo, Salanova (2010) señala que tanto los recursos estructurales como sociales, el capital psicológico positivo (autoeficacia, esperanza, optimismo, resiliencia y vinculación) y los resultados de la institución configuran la «Organización Positiva y Saludable». También, y desde otra perspectiva, el alfabetismo financiero (habilidades y conocimientos en materia financiera) y las decisiones económicas, junto con las emociones y la cultura, influyen en el bienestar económico, y por ende en la felicidad de las personas (Garay, 2015).

La contribución de Warr (2013), que emplea una metáfora vitamínica, destaca la no linealidad explicativa de fuentes de felicidad e infelicidad en el trabajo. Por una parte, separa los factores ambientales de los personales. Entre los primeros estarían "oportunidad de control, oportunidad para el uso y la adquisición de habilidades, metas generadas externamente, variedad, claridad del entorno, contacto con otros, disponibilidad de dinero, seguridad física, posición socialmente valorada, apoyo del supervisor, desarrollo de carrera y equidad" (p.100). Entre los segundos estarían: "comparaciones con otras personas, comparaciones con otras situaciones, comparaciones con otras épocas, evaluaciones de una situación relacionada con la autoeficacia, evaluación de la novedad o familiaridad, evaluaciones de relevancia personal" (p.102). Igualmente, la teoría de demanda y recursos laborales de Bakker y Demerouti (2013, como se cita en Rivas, 2015, p. 31) explica que: 
[...] las características del trabajo pueden ser organizadas en dos categorías: demanda y recursos laborales y que se pueden encontrar en casi todos los puestos de trabajo y son los desencadenantes de aspectos negativos (deterioro de la salud) y positivos (proceso motivacional). El job crafting, o los ajustes individuales de las demandas y recursos, pueden hacer el ambiente de trabajo más atractivo, menos agotador, más saludable y productivo.

También se propone el modelo teórico de experiencias positivas en el que unos factores personales criban, interpretan y valoran la percepción de los factores ambientales, recursos y demandas laborales y extralaborales, lo que influye en el grado de bienestar subjetivo (Rodríguez \& Cifre, 2012; Cifré \& Navarro, 2013).

Al igual que otros autores, el equipo de Sánchez (2018) ha investigado el concepto de «Felicidad Organizacional» encontrando factores concretos muy diferentes en las organizaciones de su muestra. Algunos de esos factores de felicidad son: preocupación de los directivos por el bienestar, estructuras flexibles, se alienta la diversidad, la democracia en las decisiones y la validez de opiniones, se comparten y valoran los logros, se dirige con justicia, transparencia y ética, el trabajo en equipo, la innovación, la comunicación, la calidad y el talento son especialmente cuidados y los factores motivacionales y de compensación son la promoción, los incentivos, la conciliación, el agradecimiento y la confianza.

Por otra parte, en el campo académico conocemos iniciativas y equipos que ahora mismo ejecutan estudios empíricos transnacionales para estudiar el «Happiness Management», que se viene definiendo como un modelo de gestión multicultural centrado en los siguientes recursos: creatividad, compromiso, innovación tecnológica, emprendimiento interno y responsabilidad social, para desarrollar en las organizaciones y en las personas la felicidad. Así se aborda en este amplio estudio tanto la felicidad organizacional, que propicia un clima laboral de innovación, comunicación constructiva y emociones positivas, como la felicidad laboral, conjunto complejo de satisfacciones y significados vitales de implicación, compromiso, confianza, cultura de equipo y desarrollo. Uno de los proyectos a los que nos referimos es el llamado «El Happiness Management, la creatividad y el bienestar en la era de la Industria 4.0 desde la perspectiva de las ciencias sociales y humanidades», que, auspiciado por la Universidad Politécnica Salesiana de Ecuador, aglutina a treinta investigadores de veinte universidades de todo el mundo. Citando a dicho proyecto, su finalidad es explorar cómo influyen -en la era de la Industria 4.0.-, los vectores de la creatividad, el desarrollo emocional y el bienestar social (calidad de vida, satisfacción, buen vivir o bien común) en los modelos de gestión y cultura organizacional orientados íntegramente a la búsqueda holística de la felicidad de su talento humano. De esta manera los máximos responsables políticos, económicos y empresariales podrán cultivar ecosistemas y tejidos productivos basados en los principios rectores de la sostenibilidad, la innovación, el know-how, la responsabilidad social, el engagement, la gestión del conocimiento y el aprendizaje colaborativo.

También en el campo organizacional, y principalmente en las áreas de desarrollo de recursos humanos y planificación estratégica, se requiere el avance investigador, puesto que diferentes estudios constatan las relaciones de la felicidad organizacional con la eficacia (Baker, Greenberg \& Hemingway, 2007). Así, Fernández (2019), utilizando como base los estudios de Carter (2017), selecciona distintas encuestas y 
estudios que, vistos en conjunto, aportan pruebas evidentes de que se hace necesario buscar esa correlación:

\section{Tabla 1. Estudios previos que demuestran correlación entre felicidad y producción laboral}

\begin{tabular}{|c|c|}
\hline $\begin{array}{l}\text { Nombre o entidad del } \\
\text { estudio }\end{array}$ & Aporte \\
\hline $\begin{array}{l}\text { Happiness and Productivity } \\
\text { (University of Warwick) }\end{array}$ & $\begin{array}{l}\text { Los empleados felices en su puesto de trabajo son un } 12 \% \\
\text { más productivos. }\end{array}$ \\
\hline $\begin{array}{l}\text { Iopener Institute } \\
\text { (Oxford University) }\end{array}$ & $\begin{array}{l}\text { Las personas con índices mayores de felicidad en su tra- } \\
\text { bajo tienen un rendimiento mayor: dedican el } 80 \% \text { de su } \\
\text { tiempo a las tareas encomendadas. En cambio, la dedica- } \\
\text { ción a las labores que deben desempeñar de las personas } \\
\text { con índices de no felicidad en el trabajo disminuye hasta el } \\
40 \% \text { de su tiempo. }\end{array}$ \\
\hline $\begin{array}{l}\text { University of California } \\
\text { (Berkeley) }\end{array}$ & Las personas felices son un $86 \%$ más creativas. \\
\hline Gallup & $\begin{array}{l}\text { Una empresa feliz aumenta un } 10 \% \text { las calificaciones de clien- } \\
\text { tes, tiene un } 55 \% \text { menos de rotación de personal, fideliza un } \\
44 \% \text { más a sus empleados, tiene un } 41 \% \text { menos de absentis- } \\
\text { mo, tiene un } 58 \% \text { menos de problemas de seguridad y tiene } \\
\text { un } 40 \% \text { menos de productos defectuosos. }\end{array}$ \\
\hline Shawn Anchor & $\begin{array}{l}\text { El trabajador feliz, motivado y satisfecho con su trabajo con- } \\
\text { sigue un aumento del } 37 \% \text { de las ventas, del } 31 \% \text { de la produc- } \\
\text { tividad y del } 19 \% \text { en la eficacia y los empleados infelices tienen } \\
\text { una media de } 15 \text { días más de baja por enfermedad que la media. }\end{array}$ \\
\hline HBR & $\begin{array}{l}\text { Un empleado feliz es un } 300 \% \text { más creativo e innovador y } \\
\text { tiene un } 125 \% \text { menos de estrés. }\end{array}$ \\
\hline Hays Group & $\begin{array}{l}\text { Las personas felices son un } 43 \% \text { más productivas y el coste } \\
\text { de reemplazar un empleado está entre el } 50 \% \text { y el } 150 \% \text { del } \\
\text { salario. }\end{array}$ \\
\hline Social Market Foundation & Los empleados felices son un $20 \%$ más productivos. \\
\hline Forbes & Una empresa feliz tiene un $66 \%$ menos de bajas laborales. \\
\hline Greenberg \& Arawaka & Una empresa feliz tiene un $31 \%$ más de productividad. \\
\hline Framingham Heart Study & $\begin{array}{l}\text { Las personas que se rodean de gente feliz tienen un } 25 \% \text { más } \\
\text { de posibilidades de imitar este estado anímico. }\end{array}$ \\
\hline
\end{tabular}

De lo que se extrae en la tabla 1, resulta meridianamente evidente que se deben buscar entornos de bienestar y productividad que den respuesta a las necesidades y cambios de la sociedad, ya no sólo por los directivos de las empresas, quienes tienen la misión de impulsar ese binomio, sino por los propios empleados y skateholders. 
Por último, aunque no es objeto de este estudio, hay que señalar que los análisis críticos con respecto al tema de la felicidad son recurrentes. Hay dos documentos que son especialmente intensos en las críticas: La felicidad paradójica de Lipovestsky (2014) y el análisis sobre la Psicología Positiva de Lazarus (2003).

Además, a pesar de las aportaciones que se realizan desde la Economía de la Felicidad, no debemos olvidar que coexisten «otras» realidades como la pobreza, la economía sumergida o el empleo precario, situaciones en las cuales el constructo «trabajo» $\mathrm{y}$ «felicidad» $\mathrm{u}$ «organizaciones felices» difícilmente tienen cabida.

Se necesita, en consecuencia, que disciplinas como la economía o la psicología, entre otras, adquieran un mayor compromiso en las soluciones de lo que no dejan de ser problemas sociales. Se debe volver a las propuestas lewinianas en las que el vínculo entre la investigación y la acción o la aplicación profesional den como resultado programas e intervenciones que propicien cambios sociales en los que se haga realidad una economía de la felicidad para todos (Yela \& Saiz, 2013). De esta manera nos podremos acercar a ese modelo de vida donde priman el bienestar subjetivo y la felicidad sin olvidar que, para que esto sea posible, se deben incluir en las agendas políticas (Morales, Nouvillas \& Arias, 2012). Todo esto además encaja con la «Economía del Bien Común» -paradigma que incluye a la Economía de la Felicidad- y el Estado del Bienestar; constructos y realidades que es preciso desarrollar aún más.

Creemos que en el desarrollo de esta área no deben desatenderse ni las dimensiones individuales -pues debe seguirse avanzando en el estudio de la felicidad personal y el bienestar subjetivo y crear instrumentos de evaluación que reflejen las dimensiones de los modelos más comprobados-, ni las dimensiones colectivas, puesto que los índices y objetivos de desarrollo relacionados con la felicidad contribuyen a la mejora de las sociedades y comunidades.

\section{Materiales y método}

Con el objetivo de constatar los intereses de los investigadores en el campo de la felicidad en el trabajo y el «Happiness Management», se ha optado en este estudio, de carácter analítico y bibliométrico, por una selección de unidades de análisis que cumplieran una serie de criterios que permitieran constatar el interés de los autores en la literatura científica de los temas seleccionados en idioma español. Para ello se han analizado documentos científicos que han sido publicados y cuyos textos se podían recuperar de las Bases de datos EDS-multidisciplinar (EBSCO Discovery Service). Este motor de búsqueda permite el acceso a bases de datos de texto completo de las publicaciones científicas de distintas áreas.

En cuanto a los términos a analizar se utilizaron las siguientes palabras claves: economía de la felicidad, felicidad en el trabajo, trabajo y felicidad y «Happiness Management». Son estos términos los principales que deben considerarse para que la búsqueda recoja de la forma más completa posible las investigaciones relacionadas con el tema de estudio. Se delimitó la búsqueda desde el año 2000 al 2018, puesto que la unidad de análisis temporal es significativa por el cambio de milenio. También se optó por seleccionar sólo el español como el idioma de la publicación. Los artículos que cumplían dichos criterios y que fueron seleccionados y analizados han sido 95, 
número que se considera suficiente para analizar en las categorías clasificatorias de los estudios bibliométricos de un término.

Los análisis se han centrado en agrupar los distintos elementos de esta unidad muestral de fuentes, que se esperan sean representativas por su forma de selección, en categorías que agrupen los estudios en las más relevantes clasificaciones para evaluar la producción científica. Así, se ha categorizado la producción relativa a los años, las áreas temáticas en las que se han centrado los estudios, el tipo de documento científico, el carácter, teórico o empírico, de los contenidos, los países de publicación y las disciplinas en las que se ha situado cada estudio.

\section{Resultados}

En primer lugar, se presenta la producción de los documentos por años. Aunque bastante desigual, se puede observar que a partir de 2011 se evidencia un aumento más o menos estable de la producción científica en este campo.

\section{Figura 1. Evolución de las publicaciones sobre felicidad y trabajo en EDS-multidisciplinar}

\section{Producción por años}

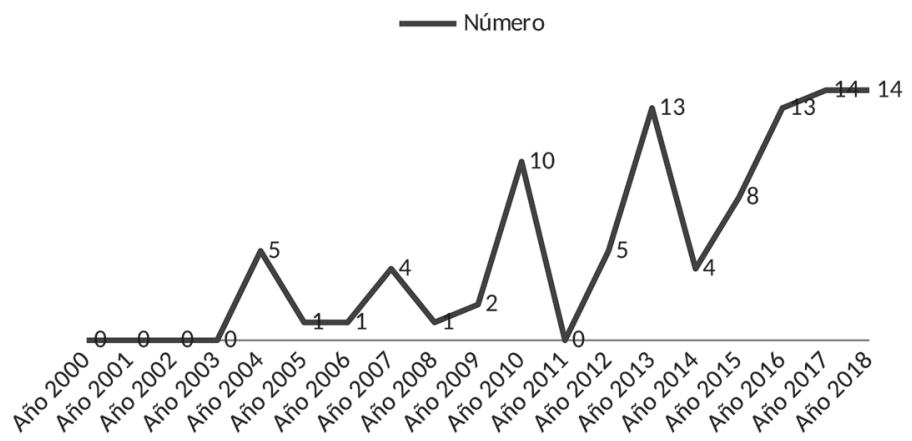

Fuente: Elaboración propia sobre datos extraídos de EDS Multidisciplinar (2019).

A continuación, se analizaron las áreas temáticas que se tratan en los artículos y, como se observa en la figura 2, se ocupan de analizar el tema de la Economía de la Felicidad unido en su mayoría a diversas variables relacionadas con el trabajo y el desarrollo económico. Las dos áreas que más se han estudiado en la producción científica de estos años son el de la felicidad en el trabajo (18\%) y el de la Economía de la Felicidad (17\%), correspondiendo en su mayoría a abordajes teóricos. Seguidamente las áreas más estudiadas se corresponden a temas relacionados con el mundo del trabajo: liderazgo y felicidad (8\%), empresas felices $(8 \%)$ y organizaciones sanas $(7 \%)$. Seguidamente aparecen un grupo de documentos que tienen que ver con variables sociodemográficas estudiadas en relación a la Economía de la Felicidad (5\%) y 
en la misma medida a estudios que analizan el crecimiento económico, las escalas de medida de felicidad, la motivación laboral o estudios que tienen que ver con los antecedentes históricos de este tema. De forma más minoritaria (3\%) se presentan otros trabajos que analizan desde diversas ópticas el tema de la felicidad, como son el ocio y el trabajo, la pobreza o el burnout profesional. Además aparecen estudios críticos sobre este tema abordado desde el hiperconsumo (2\%). Por último, hay un pequeño grupo de documentos que examinan diversos aspectos como la influencia de la vivienda, el éxito profesional, las políticas sociales y laborales y los valores (1\%).

Figura 2. Temáticas centrales de los artículos emergentes en EDS-multidisciplinar

\section{Areas temáticas}

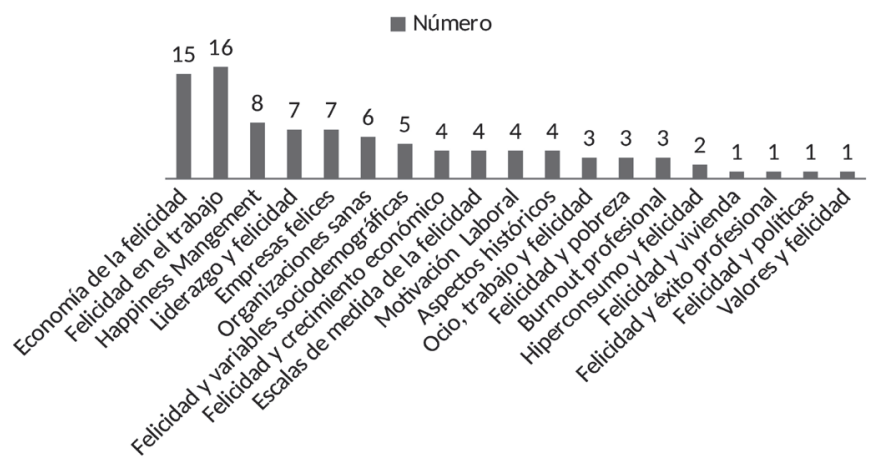

Fuente: Elaboración propia sobre datos extraídos de EDS Multidisciplinar (2019).

Por otra parte, se presentan los tipos de documentos científicos en los que se han abordado los temas objeto del presente estudio, siendo mayoritariamente el artículo de revista ( $86 \%$ ) el formato preferido para presentar estos tipos de estudios. Muy de lejos en el abordaje de esta temática aparecen las tesis doctorales, los libros, capítulos de libros y ponencias como puede observarse en la figura 3.

Figura 3. Tipo de soporte/formato de los documentos emergentes EDS-multidisciplinar Tipo de documento

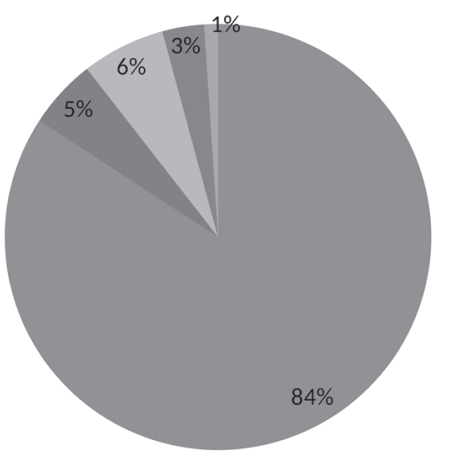

Artículo de revista

- Tesis doctoral

Libro

- Capítulo de libro

- Ponencia 
Si examinamos las disciplinas a las que pertenecen los artículos de las revistas analizadas nos encontramos que la mayor parte de las publicaciones se reparten entre las áreas de la economía y la empresa $(40 \%)$ y la psicología (28\%), y más alejadas en cuanto a número, revistas que pertenecen a los recursos humanos, la historia, la educación u otras muy minoritarias (política, salud, etc.). De esto se colige con meridiana claridad el carácter transdisciplinar que tiene el tema de la felicidad y el «Happiness Management» dentro de las Ciencias Sociales. En cuanto al carácter de estos documentos se analizaron si habían sido abordajes teóricos o investigación empírica sobre el tema. Los resultados después del análisis fueron que el 59\% de los documentos emergentes son análisis teóricos de la cuestión y el 41\% investigaciones empíricas.

Dentro de la investigación empírica, los trabajos se desarrollan al 50\% en América Latina (México, Chile, Colombia, Bolivia, Brasil, Argentina y Venezuela) y el otro 50\% en España -recordando que el criterio de búsqueda se hizo en idioma español-. Aunque en los primeros la distribución resulta muy dispersa, es Brasil, con seis trabajos, el país que más tiene; seguido de Argentina, Chile y Colombia con dos y México, Bolivia y Venezuela con uno. Por otra parte, hay dos trabajos en los que se referencia América Latina en su conjunto sin señalar país.

\section{Conclusiones y discusión}

Con estos resultados pueden hacerse algunas interpretaciones claras, puesto que el desglose y las relaciones que estos datos tienen, pueden ser relevantes para evaluar los intereses y líneas de avance de las investigaciones.

La tendencia claramente creciente de la producción científica sobre felicidad y trabajo tiene dos vertientes importantes: Por un lado, el propio tiempo de grandes cambios, que hace surgir continuamente nuevos paradigmas explicativos y prescriptivos. En este sentido la «Felicidad Organizacional» puede ser una nueva conceptualización que recoge los constructos antiguos de motivación, satisfacción, calidad de vida laboral, engagement, pasión por el trabajo, bienestar subjetivo, etc. Y, por otro lado, coincide este incremento con el fin de la crisis económica. Superar y dejar atrás las preocupaciones de este gran desequilibrio del sistema económico mundial, ha vuelto a centrar el interés por hacer más productivas y humanas las empresas, preservando la salud laboral y mejorando el desarrollo personal (Peiró, 2011; Ramos \& Peiró, 2014).

La revisión de las áreas temáticas sitúa la mayoría de los estudios en el núcleo de los modelos que tratan la economía, el trabajo y la felicidad. Ello es obvio, puesto que cabe pensar que los investigadores tratan los aspectos generales de una manera mayoritaria, tanto en su aspecto econométrico, que siempre ha mantenido el interés por las comparativas de los distintos índices entre países (Rivas et al., 2015), como el relacionado con el trabajo (Rodríguez \& Cifre, 2012).

Pero a pesar de ese dato más general, a continuación, se observa que se han tratado temas que son más concretos. En el siguiente lugar era muy de esperar que se tratara el liderazgo y sus relaciones con la felicidad. Este creciente interés por el «Happiness Management» recoge el avance en los estudios del liderazgo transformacional, emocional, positivo, auténtico, servidor que eran tendencia en las investigaciones del liderazgo positivo (Yukl, 2008; Sánchez, 2010; Blanch et al., 2016). Al ser el 
liderazgo el aspecto psicosocial más estratégico en las organizaciones, su optimización es muy visible. Incluso algunas compañías han dejado atrás las denominaciones de Jefe de Personal, Director de Recursos Humanos o Director de Personas cambiándolas por la de Director de Felicidad (por ejemplo, Open English). Muchos estudios actuales están trabajando este constructo (Ravina, Villena \& Gutiérrez, 2017; Ravina, Tobar \& Marchena, 2019).

Al tema del liderazgo le siguen dos categorías que tienen que ver con anteriores intereses, como son las empresas felices y las organizaciones saludables y positivas, que constituye el ideal del funcionamiento óptimo de la salud de las personas y las organizaciones y la gestión efectiva de su bienestar y desarrollo (Lorente \& Vera, 2010; Salanova, 2010; Salanova et al., 2017; Rivera et al., 2018). Y en los siguientes lugares aparecen las relaciones de los descriptores estudiados con aspectos sociológicos (demografía, crecimiento económico, historia, ocio, pobreza, vivienda, política).

También hay propuestas de escalas de medida y que no son nada frecuentes de encontrar. A pesar de la propuesta de algunos instrumentos de medida específica de felicidad en el trabajo (Salas, Alegre \& Fernández, 2013) se hace necesario ahondar la investigación en esta área para la construcción de estas herramientas.

Por otra parte, los documentos abordan otros aspectos psicosociales como son la motivación, el éxito y los valores. En conjunto, las variables que se analizan en los diversos documentos estudiados son las que tienen impacto sobre el concepto de felicidad aplicado al mundo laboral tanto en su vertiente positiva como negativa.

Además de lo anteriormente comentado, algunos de los temas tratados en estos documentos ponen de manifiesto la importancia del bienestar subjetivo de las personas en relación al mundo laboral, la satisfacción personal y la influencia negativa de variables que están interfiriendo en esto. Los artículos seleccionados tratan todas aquellas dimensiones que efectivamente tienen que ver con esto: variables demográficas, sociales y económicas, salud y aquellas que tienen que ver con la conducta y la situación vital de las propias personas.

En cuanto a los resultados del tipo de documento científico, como era de esperar es mayoritario el artículo de revista. Sólo se han indexado cinco tesis doctorales, tres libros y tres capítulos de libro. Esto es muy conveniente, puesto que la difusión de las revistas y su alcance global, pueden ser mayores. Es necesario también la utilización de los metaanálisis comparativos en este campo que compendien los estudios globalmente y nos muestren la efectividad de los diversos modelos que procuren afectos positivos en el trabajo.

Separadamente se ha analizado el carácter del documento, que mayoritariamente refleja los estudios teóricos del tema en este período. Se hace necesario incidir más en resultados de investigación empírica que favorezca la aparición de evidencias y sirvan para la construcción de organizaciones y modelos laborales sanos. Estos sí que pueden sentar las bases de los modelos importantes, como se ha expuesto antes, pero esto debe verse acompañado de estudios que reflejen la realidad. Algunos de ellos seguro que están haciéndose actualmente.

Las disciplinas a las que pertenecen las revistas en las que se han realizado las publicaciones analizadas reflejan el carácter bifronte del tema, económico y psicológico en cuanto reflejo del bienestar de las personas. Se hace necesaria la 
aportación de la psicología, no sólo desde sus áreas laborales sino también desde otras áreas como la psicología económica (Billón, 2002; Bonavia \& Quintanilla, 2005; Quintanilla, 2010); ésta puede añadir el conocimiento sobre la conducta económica de las personas como productora de actitudes positivas o negativas en su ámbito laboral. Los factores económicos indudablemente tienen una influencia central en la conducta de las personas no sólo en su ámbito privado sino también en su esfera pública y por ende en su vida laboral.

En cuanto a las líneas futuras de investigación nos encontramos en un momento de revisión de los modelos, al que contribuye nuestro estudio, y constatamos tanto en nuestros entornos académicos como organizacionales el amplio interés del tema y, por tanto, la necesidad de un abordaje multidisciplinar, global y científico que permita contrastar evidencias claras de la eficacia de su aplicación.

Por tanto, se hace necesario el abordaje multidisciplinar del tema para encontrar caminos no solo del crecimiento económico, sino para que este contribuya a la prosperidad de las personas en todas sus dimensiones.

\section{Referencias}

De Barbieri, A. (2018). Economía de la felicidad: claves para vencer el cansancio y vivir con sentido. Madrid, España: Grijalbo.

Baker, D., Greenberg, C., \& Hemingway, C. (2007). Empresas Felices=Empresas Rentables. Barcelona, España: Gestión 2000.

Bakker, A.B., Rodríguez-Muñoz, A., \& Derks, D. (2012). La emergencia de la Psicología de la Salud Ocupacional Positiva. Psicothema, 24(1), 66-72.

Bakker, A. B., \& Demerouti, E. (2013). La teoría de las demandas y los recursos laborales. Revista de Psicología del Trabajo y de las Organizaciones, 29(3), 107-115. https://doi.org/10.5093/tr2013a16

Billón, M. (2002). Psicología y economía desde una perspectiva interdisciplinar. Encuentros multidisciplinares, 4(11), 2-10. http://hdl.handle.net/10486/668582

Blanch, J., Gil, F., Antino, M. \& Rodríguez Muños, A. (2016). Modelos de liderazgo positivo: marco teórico y líneas de investigación. Papeles del Psicólogo, 37(3), 170-176.

Bonavía, T., \& Quintanilla, I. (2005). Psicología y economía. Valencia, España: Universidad de Valencia.

Carter, B. (2017). 2017 Employee, Engagement $\mathcal{E}$ Loyalty statistics, Access Perks, 28 de agosto. Recuperado de http://bit.ly/2Xqd5VG

Castells, M. (2008). La sociedad red. Madrid, España: Alianza editorial.

Cifré, E., \& Navarro, $M^{\mathrm{a}}$ L. (2013). Construyendo puentes entre la Responsabilidad Social Empresarial y la Salud Psicosocial en las Organizaciones: una Guía 2.0 para el incremento de la Empleabilidad. Estudios financieros. Revista de Trabajo y Seguridad Social: Comentarios, casos prácticos: recursos humanos, 359, 163-204. http://hdl.handle.net/10234/93650

De los Ríos, A.L. (2016). Felicidad y economía: la felicidad como utilidad en la economía. Equidad EB Desarrollo (26), 115-143. https://doi.org/10.19052/ed.3700

Diener, E. (2000). Subjective well-being: The science of happiness and a proposal for a national index. American Psychological, 55(1), 34-43. https://psycnet.apa.org/doi/10.1037/0003066X.55.1.34

Fernández, Y. (2019). ¿Es rentable invertir en felicidad?, Finsi, 6 de febrero. Recuperado de http://bit. ly/2QCUIdD

Frey, B. (2018). Economics of Happiness. Cham, Switzerland: Springer. https://doi.org/10.1007/9783-319-75807-7 
Galiano Coronil, A., \& Ravina Ripoll, R. (2015). Un mapa de la felicidad de los titulados universitarios españoles, en el adiós de la crisis económica (2014). Oikos: Revista de la Escuela de Administración y Economía, 19(40), 123-136.

Garay, G. (2015). Las Finanzas Conductuales, el Alfabetismo Financiero y su Impacto en la Toma de Decisiones Financieras, el Bienestar Económico y la Felicidad. Revista Perspectivas, (36), 7-34.

Lazarus, R. S. (2003). Does the positive psychology movement have legs? Psychological Inquiry, 14(2), 93-109. https://psycnet.apa.org/doi/10.1207/S15327965PLI1402_02

Lipovestsky, G. (2014). La felicidad paradójica. Barcelona, España: Anagrama.

Lorente, L., \& Vera, M. (2010). Las organizaciones saludables: "El engagement en el trabajo". Gestión práctica de riesgos laborales: Integración y desarrollo de la gestión de la prevención, 73, pp. 16-20.

Moccia, S. (2016). Felicidad en el trabajo. Papeles del psicólogo, 37(2), 143-151.

Morales, J.F., Nouvillas, E. \& Arias, A.V. (2012). Aplicaciones de la psicología social. En A.V. Arias, J. F. Morales, E. Nouvillas \& J.L.Martínez-Rubio (Coords.). Psicología Social Aplicada (pp.1-43). Madrid, España: Médica Panamericana.

Peiró, J. M. (2011). La flexibilidad laboral en un entorno de crisis económica. Contribuciones desde la psicología. Infocop, 55, 14-18.

Popescu, G.H. (2016). Does economic growth bring about increased happiness? Journal of Self-Governance and Management Economics, 4(4), 27-33. https://doi.org/10.22381/JSME4420163

Quintanilla, I. (2010). La psicología económica y del consumidor en la sociedad de la complejidad y la incertidumbre. Informació psicológica, 100, 115-118.

Ramos, J. \& Peiró, J.M. (2014). La Psicología del trabajo y las organizaciones en tiempos de crisis económica. Papeles del psicólogo, 35(1), 1-4.

Ravina, R., Tobar, L. B., \& Marchena, J. (Eds.). (2019). Happiness Management: A Lighthouse for Social Wellbeing, Creativity and Sustainability. Switzerland: Peter Lang CH. https://doi.org/10.3726/ b15813

Ravina, R., Villena, F., \& Gutiérrez, G.A. (2017). Una aproximación teórica para mejorar los resultados de innovación en las empresas desde la perspectiva del "Happiness Management". Retos, Revista de Ciencias de la Administración y Economía, 14(7), 113-129. https://doi. org/10.17163/ret.n14.2017.06

Rivas, L.A. (Coord.) (2015). Sectores de la nueva economía 20+20. Economía de la Felicidad. Madrid, España: EOI.

Rivera, D.A., Carrillo, S.M., Forgiony, F., Nuván, I.L., \& Rozo, A.C. (2018). Cultura organizacional, retos y desafíos para las organizaciones saludables. Revista Espacios, 38, 1-14. http:// hdl.handle.net/20.500.12442/2165

Rodríguez, A.M., \& Cifre, E. (2012). Flow y bienestar subjetivo en el trabajo. Madrid, España: Síntesis.

Rodríguez-Muñoz, A., \& Sanz-Vergel, A.I. (2011). La felicidad y el bienestar en el trabajo. Mente y cerebro, 50, 42-49.

Retzbach, J. (2018). La felicidad se construye con sentido. Mente y Cerebro, 90, 44-49.

Salanova, M. (2010). Psicología de la salud ocupacional. Madrid, España: Síntesis.

Salanova, M. (2010). Organizaciones saludables, organizaciones resilientes. Gestión Práctica de Riesgos Laborales, 58, 18-23. http://hdl.handle.net/10234/73232

Salanova, M., Llorens, S., Cifre, E., \& Martínez, I. M. (2012). We need a hero! Toward a validation of the healthy and resilient organization (HERO) model. Group $\mathcal{E}$ Organization Management, 37(6), 785-822. http://dx.doi.org/10.1177/1059601112470405

Salas Vallina, A., Alegre, J. \& Fernández, R. (2013). La medición de la felicidad en el trabajo y sus antecedentes : un estudio empírico en el área de alergología de los hospitales públicos españoles. Trabajo: Revista andaluza de relaciones laborales, 28, 83-116.

Sánchez, J. F. (2010). Liderazgo: teorías y aplicaciones. Salamanca, España: Universidad Pontificia de Salamanca.

Sánchez, J.F. (2018). El significado vital en las organizaciones: aportaciones de la economía de la 
felicidad. Cauriensia, 13, 143-156. https://doi.org/10.17398/2340-4256.13.143

Seligman, M. (2011). La vida que florece. Barcelona, España: Ediciones B.

Thivissen, P. (2018). La importancia del sentido de la vida. Mente y Cerebro, 90, 50-55.

Vera, B. (2006). Psicología Positiva: Una nueva forma de entender la psicología. Papeles del psicólogo, 27(1), 3-8.

Vera, B. (2008). Psicología Positiva. Una nueva forma de entender la psicología. Madrid, España: Calamar Ediciones.

Warr, P. (2013). Fuentes de felicidad e infelicidad en el trabajo: una perspectiva combinada. Journal of Work and Organizational Psychology, 29(3), 99-106. http://dx.doi.org/10.5093/tr2013a15

Yela García, C. y Saiz Galdós, J. (2013). Hacia una psicología social de los problemas sociales (I): psicología social aplicada y problemas sociales. En C. Yela García (Coord.), Psicología social de los problemas sociales (pp.41-85). Madrid, España: Grupo 5.

Yukl, G., (2008). Liderazgo en las organizaciones. Madrid, España: Pearson. 